\title{
DUAS DÉCADAS DE QUÍMICA NOVA
}

Helena M. C. Ferraz e Vera L. Pardini

Instituto de Química - Universidade de São Paulo - CP 26077 - 05599-970 - São Paulo-SP

\begin{abstract}
TWO DECADES OF QUIMICA NOVA. A brief overview of the 20 years of Química Nova, a journal published by the Sociedade Brasileira de Química, is presented.
\end{abstract}

\author{
Keywords: Química Nova; Brazilian Chemical Society; chemistry in Brazil.
}

Como parte das comemorações dos 20 anos da SBQ, sua Diretoria decidiu publicar um número especial de Química Nova e nos convidou para que escrevêssemos sobre a trajetória da revista ao longo destes anos.

Em um artigo publicado em $1992^{1}$, já havíamos apresentado um levantamento dos primeiros 14 anos de Química Nova; desta maneira, o presente trabalho constitui uma atualização do anterior, englobando, desta feita, duas décadas de existência da revista.

A idéia de editar uma revista que fosse o órgão oficial de divulgação da Sociedade Brasileira de Química (SBQ) surgiu logo após a criação da Sociedade, em julho de 1977, e foi concretizada logo a seguir, por iniciativa do Prof. Eduardo M. A. Peixoto. O primeiro número de Química Nova, com uma tiragem de 1500 exemplares, foi lançado em janeiro de 1978 - portanto, um semestre após a criação da SBQ - e trazia em sua capa a reprodução da Ata da Assembléia Geral de Fundação da Sociedade.

De 1978 até 1992, foram editados periodicamente, às vezes com um atraso de muitos meses, às vezes quase que em dia, 15 volumes de Química Nova, com publicação trimestral, perfazendo o total de 60 números. A partir de 1993, devido ao significativo aumento de trabalhos submetidos desde 1991, a editoria decidiu mudar a periodicidade da revista, que passou a sair bimestralmente.

Ainda em 1978, Química Nova já estava indexada pelo Atomindex e, a partir de 1980, pelos serviços do Chemical Abstracts, sendo que, desde 1995, encontra-se também indexada na base de dados de Química do Citation Index (ISI).

Sempre visando ao seu objetivo primeiro, que é o de divulgar trabalhos de pesquisa em química, a revista foi se aperfeiçoando, modificando algumas de suas seções, passando pelas mãos de vários editores, mas sempre mantendo um perfil que lhe é característico até hoje.

Eduardo Peixoto editou a revista durante 4 anos, de 1978 a 1981, contando com a colaboração de um Conselho Editorial, composto de 12 especialistas de notório saber em suas respectivas áreas.

Em janeiro de 1982, Fernando Galembeck assumiu como editor interino e, a partir de julho do mesmo ano, como editor, cargo que ocupou até abril de 1984. Ainda em 1982, a revista passou a contar, além do Conselho Editorial, com uma Assessoria composta de 6 membros, representando as principais áreas em que a Química é tradicionalmente dividida, e que auxiliavam mais de perto o editor.

Datam deste período as primeiras modificações na política editorial da revista. Até 1983, Química Nova publicava exclusivamente trabalhos em português, situados em uma das seguintes categorias:

Novidades Científicas - trabalhos curtos, contendo a essência de um trabalho recém publicado, ou aceito para publicação em revistas especializadas;

Artigos - trabalhos inéditos de revisão, de caráter educacional;
Notas técnicas - trabalhos originais sobre novas técnicas de análise, síntese, cálculo, educação ou construção de novos aparelhos e de acessórios, etc.;

Assuntos Gerais - assuntos de interesse da comunidade e que não se adaptavam às outras seções;

Notas Sociais - informações tais como contratações de docentes, visitas de professores, congressos, etc.;

Cartas ao Editor.

Em abril de 1993, por iniciativa dos membros da Assessoria Editorial, foi criada a seção de Comunicações, destinada à divulgação de resultados preliminares de pesquisa. Considerando que Química Nova já estava indexada pelos serviços do Chemical Abstracts, esta nova seção não somente garantiria a originalidade dos estudos nela relatados, como também ofereceria mais um espaço para a publicação de resultados inéditos de pesquisa.

Com a saída de Fernando Galembeck da editoria, em 1984, tomou posse João V. Comasseto, que exerceu o cargo até o final do mesmo ano.

A partir de janeiro de 1985 teve início, então, uma segunda fase de transição da revista: Química Nova passou a ser editada não mais em São Paulo, e sim em Campinas, de início novamente sob a responsabilidade de Fernando Galembeck e, logo em seguida, já com um novo corpo editorial.

Assim, de julho de 1985 até janeiro de 1989, Química Nova teve como editores Eduardo J. S. Vichi, Aécio P. Chagas e Yoshitaka Gushikem, todos da UNICAMP. Os antigos Conselho Editorial e Assessoria Editorial foram substituídos pelo que se convencionou chamar de Corpo Editorial, abrangendo um número maior de pesquisadores e atendendo a um critério mais amplo de representatividade por regiões do país.

Foram efetuadas, também, algumas modificações na linha editorial da revista, que passou a contar com novas normas de publicação em janeiro de 1986. As principais mudanças constaram da extinção da seção de Novidades Científicas e da subdivisão da seção de Artigos em: Artigos Originais, Artigos de Revisão, Artigos de Divulgação e Artigos sobre Educação. As Notas Sociais, que provavelmente foram o embrião do Boletim Informativo da SBQ, já haviam sido abolidas há algum tempo.

Passaram a ser aceitos trabalhos redigidos em inglês - além das comunicações - e também em espanhol.

Em abril de 1989, a revista voltou a ser editada em São Paulo, contando com três editores associados - Helena M. C. Ferraz, Vera L. Pardini e João V. Comasseto - sendo este último substituído, a partir de 1992, por Angelo da C. Pinto. Em janeiro de 1995, Helena Ferraz e Angelo Pinto cederam sua vez para Eliezer J. de L. Barreiro e Joel C. Rubim. Desde setembro de 1996 a revista conta com dois editores associados - Vera Pardini e Eliezer Barreiro.

Em janeiro de 1990, em virtude do lançamento do Journal of the Brazilian Chemical Society, a editoria decidiu efetuar, novamente, algumas modificações na política editorial da revista. Assim, foi abolida a seção de Comunicações e estabeleceu-se 
que todos os trabalhos, com exceção dos Artigos Originais, deveriam ser redigidos em português (ou, eventualmente em espanhol). Atualmente, devido à demanda, a editoria decidiu voltar a publicar nas três línguas, português, espanhol e inglês.

De uma maneira bastante resumida, é esta a trajetória editorial de Química Nova. As tabelas e os gráficos que se encontram a seguir são ilustrativos das atividades e da evolução da revista nestes 20 anos.

Na tabela 1 é mostrada a distribuição dos trabalhos dentro de cada modalidade, evidenciando um significativo aumento do número de artigos de revisão e divulgação nos últimos anos.

A tabela 2 mostra que houve um aumento acentuado do número de trabalhos submetidos para publicação em 1991, tendência esta que vem se mantendo até hoje (figura 1).

Os dados da tabela 2 indicam, também, que está havendo um aumento progressivo no número de trabalhos recusados, demonstrando assim um aprimoramento na prática de avaliação de trabalhos pelos pares. As figuras 1 e 2 complementam esta tabela.
Nas tabelas 3 e 5 fez-se um apanhado geral da contribuição de cada estado e do exterior, respectivamente, durante os 20 anos da revista. É necessário explicar, nesse ponto, o critério adotado para a verificação da origem do trabalho: para um mesmo artigo foram considerados os endereços de todos os autores. Desta maneira o total apresentado por estado ou país, nestas tabelas, é bem maior que o número real de trabalhos publicados na revista (vide tabela 1). O mesmo critério foi adotado na elaboração das tabelas 4 e 6 .

A figura 3 complementa a tabela 3 , sendo que foram considerados somente os estados que publicaram no mínimo quatro trabalhos.

A tabela 4 revela que as instituições que mais contribuíram foram USP, UNICAMP e UFRJ. Entretanto, deve-se ressaltar que a contribuição da USP e da UFRJ é um somatório dos trabalhos de várias de suas unidades, como pode ser visto na tabela 6, ao passo que a da UNICAMP refere-se, em sua grande maioria, ao Instituto de Química.

Tabela 1. Distribuição dos trabalhos publicados por modalidade.

\begin{tabular}{|c|c|c|c|c|c|c|c|c|c|}
\hline \multirow[t]{2}{*}{ Ano } & \multicolumn{8}{|c|}{ Modalidade } & \multirow[t]{2}{*}{ Total } \\
\hline & Art. & Rev. & Div. & N.T. & Ed. & A.G. & N.C. & Com. & \\
\hline 1978 & 9 & - & - & 10 & - & 2 & 12 & - & 33 \\
\hline 1979 & 10 & - & - & 10 & - & 6 & 6 & - & 32 \\
\hline 1980 & 5 & - & - & 7 & 5 & 3 & 8 & - & 28 \\
\hline 1981 & 3 & - & - & 7 & 4 & 4 & 7 & - & 25 \\
\hline 1982 & 5 & - & - & 2 & 5 & 6 & 10 & - & 28 \\
\hline 1983 & 9 & - & - & 5 & 3 & 4 & 9 & 11 & 41 \\
\hline 1984 & 19 & - & - & 6 & 7 & 3 & 5 & 12 & 52 \\
\hline 1985 & 20 & 8 & - & 3 & 7 & 3 & 5 & 15 & 61 \\
\hline 1986 & 17 & 5 & 1 & 3 & 9 & 2 & - & 13 & 50 \\
\hline 1987 & 24 & 2 & 5 & 8 & 4 & 4 & - & 11 & 58 \\
\hline 1988 & 49 & 3 & 5 & 5 & 11 & 1 & - & 8 & 82 \\
\hline 1989 & 28 & 5 & 6 & 9 & 10 & 5 & - & 9 & 72 \\
\hline 1990 & 37 & 2 & 5 & 5 & 3 & 4 & - & 5 & 61 \\
\hline 1991 & 18 & 4 & 11 & 11 & 9 & 4 & - & - & 57 \\
\hline 1992 & 26 & 9 & 6 & 7 & 8 & 5 & - & - & 61 \\
\hline 1993 & 59 & 11 & 9 & 4 & 10 & 11 & - & - & 104 \\
\hline 1994 & 52 & 4 & 9 & 8 & 5 & 5 & - & - & 83 \\
\hline 1995 & 36 & 12 & 16 & 9 & 7 & 13 & - & - & 93 \\
\hline 1996 & 40 & 21 & 17 & 13 & 8 & 6 & - & - & 105 \\
\hline 1997 & 37 & 19 & 18 & 8 & 10 & 6 & - & - & 98 \\
\hline
\end{tabular}

Art. - Artigo; Rev. - Revisão; Div. - Divulgação; N.T. -Nota técnica; Ed. - Educação; A.G. - Assuntos Gerais; N.C. - Novidades Científicas; Com. - Comunicações. 
Tabela 2. Destino dos trabalhos submetidos para a publicação (1985 - 1997).

\begin{tabular}{|c|c|c|c|c|c|c|c|c|c|c|c|c|c|c|}
\hline \multirow[t]{2}{*}{ Trabalhos } & \multirow[b]{2}{*}{85} & \multicolumn{12}{|c|}{ Ano } & \multirow{2}{*}{ Total } \\
\hline & & 86 & 87 & 88 & 89 & 90 & 91 & 92 & 93 & 94 & 95 & 96 & 97 & \\
\hline Submetidos & 45 & 60 & $105^{\mathrm{a}}$ & 84 & 68 & $98^{b}$ & 102 & $107^{\mathrm{c}}$ & $129^{\mathrm{d}}$ & 137 & 150 & $191^{\mathrm{e}}$ & $163^{\mathrm{f}}$ & 1439 \\
\hline Aceitos & 33 & 45 & 88 & 72 & 50 & 72 & 73 & 82 & 98 & 99 & 119 & 117 & $52^{\mathrm{f}}$ & 1000 \\
\hline Recusados & 7 & 5 & 4 & 5 & 10 & 15 & 21 & 15 & 19 & 33 & 26 & 39 & $20^{\mathrm{f}}$ & 219 \\
\hline Retirados & 5 & 10 & 13 & 7 & 8 & 11 & 8 & 10 & 12 & 5 & 5 & 13 & $1^{\mathrm{f}}$ & 108 \\
\hline Em andamento & - & - & - & - & - & - & - & - & - & - & - & $22^{\mathrm{f}}$ & $90^{\mathrm{f}}$ & 112 \\
\hline
\end{tabular}

a Sendo 23 encomendados para a revista em homenagem ao Prof. Ricardo Ferreira.

b Sendo 26 encomendados para a revista em homenagem ao Prof. Otto Gottlieb.

c Sendo 10 encomendados para a revista da Eco-92.

d Sendo 23 encomendados para a revista em homenagem ao Prof. Giusepe Cilento.

e Sendo 20 submetidos para o número especial relativo ao II Simpósio Nacional de Vidros.

f Dados até Outubro/97.

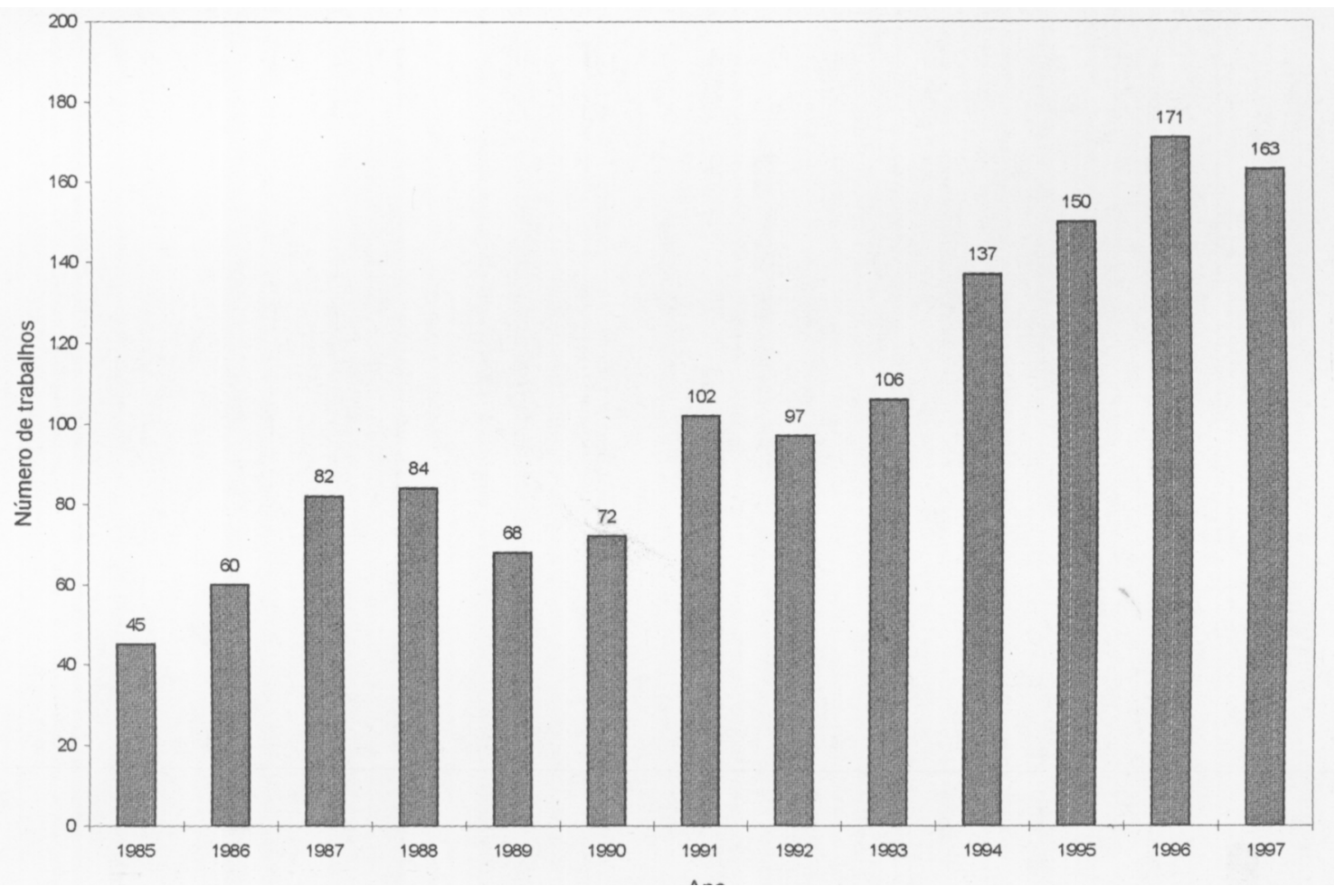

Figura 1. Número de trabalhos submetidos para publicação (1985-1997), (1997-dados até outubro; no fechamento da revista, em novembro, este número era 183).

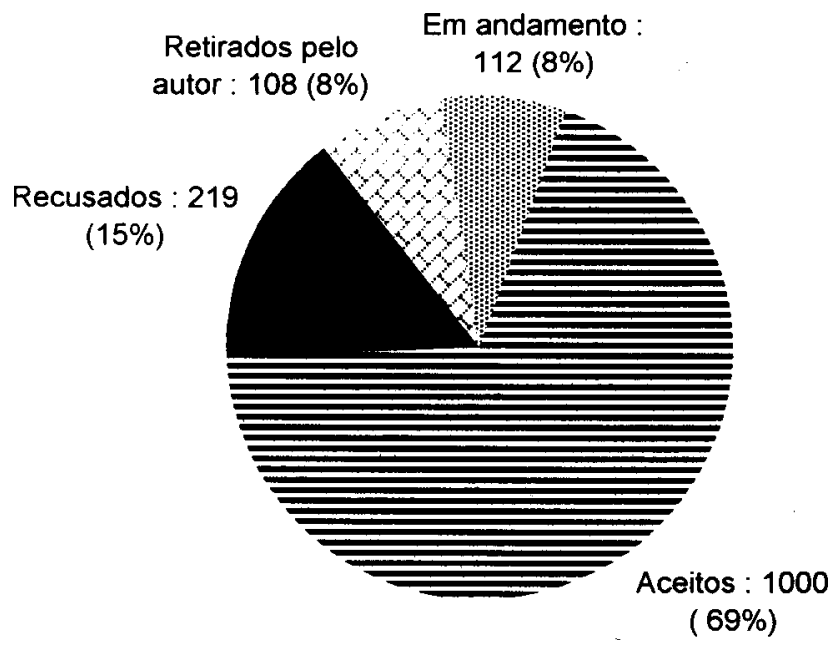

Figura 2. Destino dos trabalhos submetidos para publicação (1985-1997). 
Tabela 3. Distribuição dos trabalhos publicados por origem: Estados.

\begin{tabular}{|c|c|c|c|c|c|c|c|c|c|c|c|c|c|c|c|c|c|c|c|c|c|}
\hline $\begin{array}{l}\text { Ano } \\
\text { Est. }\end{array}$ & 78 & 79 & 80 & 81 & 82 & 83 & 84 & 85 & 86 & 87 & 88 & 89 & 90 & 91 & 92 & 93 & 94 & 95 & 96 & 97 & Total \\
\hline SP & 27 & 21 & 16 & 17 & 14 & 27 & 28 & 24 & 26 & 38 & 38 & 40 & 38 & 40 & 49 & 48 & 41 & 48 & 54 & 49 & 683 \\
\hline RJ & 5 & 5 & 3 & 5 & 6 & 7 & 13 & 24 & 20 & 20 & 22 & 17 & 16 & 11 & 15 & 5 & 14 & 24 & 25 & 27 & 284 \\
\hline MG & 1 & 3 & 3 & & 2 & 3 & 3 & 1 & 3 & 10 & 10 & 8 & 5 & 1 & 9 & 23 & 6 & 11 & 16 & 118 & \\
\hline $\mathrm{RS}$ & & 3 & 1 & 2 & & 1 & & 1 & & 2 & 3 & 2 & 1 & 1 & 2 & 4 & 4 & 2 & 8 & 5 & 42 \\
\hline $\mathrm{PE}$ & 4 & & 2 & 2 & 1 & 2 & 2 & 2 & & 1 & 9 & 1 & & 2 & & & 4 & 3 & 4 & 2 & 41 \\
\hline $\mathrm{CE}$ & 1 & 2 & & & & 1 & 2 & 6 & 5 & 4 & 2 & 6 & 1 & & 1 & 4 & 2 & 1 & 1 & & 39 \\
\hline $\mathrm{SC}$ & & & & & & & & & 1 & 1 & 2 & & 1 & 1 & 2 & 2 & 4 & 3 & 6 & 6 & 29 \\
\hline PR & & & 1 & & 1 & & & 1 & & & 1 & 1 & 2 & 2 & 3 & 5 & 1 & 4 & 3 & 3 & 28 \\
\hline DF & & 2 & & 1 & 1 & 1 & 1 & 2 & 1 & 1 & 1 & 1 & & 2 & 1 & 2 & 2 & 2 & 1 & 3 & 25 \\
\hline BA & & & & & & & 1 & 1 & 2 & 1 & & 1 & 1 & & 2 & 2 & 1 & 3 & 4 & 3 & 22 \\
\hline PB & & & & & & & & & 2 & & & 3 & 1 & 3 & & 3 & 1 & 1 & 2 & 3 & 19 \\
\hline RN & & & 1 & & & 2 & 3 & 1 & 2 & 1 & & 1 & & & & & 1 & 1 & 1 & & 14 \\
\hline $\mathrm{AM}$ & & & & & & 1 & & & & 1 & & & 3 & & & & & 1 & & & 6 \\
\hline $\mathrm{AL}$ & & & & & 2 & & & & 1 & & & & & & & 1 & & & & 1 & 5 \\
\hline $\mathrm{ES}$ & & & & & & & & & & & & & & & & 1 & 1 & & 1 & 1 & 4 \\
\hline $\mathrm{PA}$ & & & & & & & & & & 2 & & & 2 & & & & & & & & 4 \\
\hline MS & & & & & & & & & & & & & & & 1 & & 1 & & & 2 & 4 \\
\hline MT & & & & & & & 1 & & & & & & & & 1 & & 1 & & & & 3 \\
\hline PI & & & & & & & & & & 1 & & & & & & & & & 1 & 1 & 3 \\
\hline $\mathrm{RR}$ & & & & & & & & & & & & & & & & & & & 1 & 1 & 2 \\
\hline SE & & & & & & & & & & & 1 & & & & & & & 1 & & & 2 \\
\hline RO & & & & & & & & & & & & & & & & & & & 1 & & 1 \\
\hline GO & & & & & & & & & & & & & & & & & & 1 & & & 1 \\
\hline
\end{tabular}

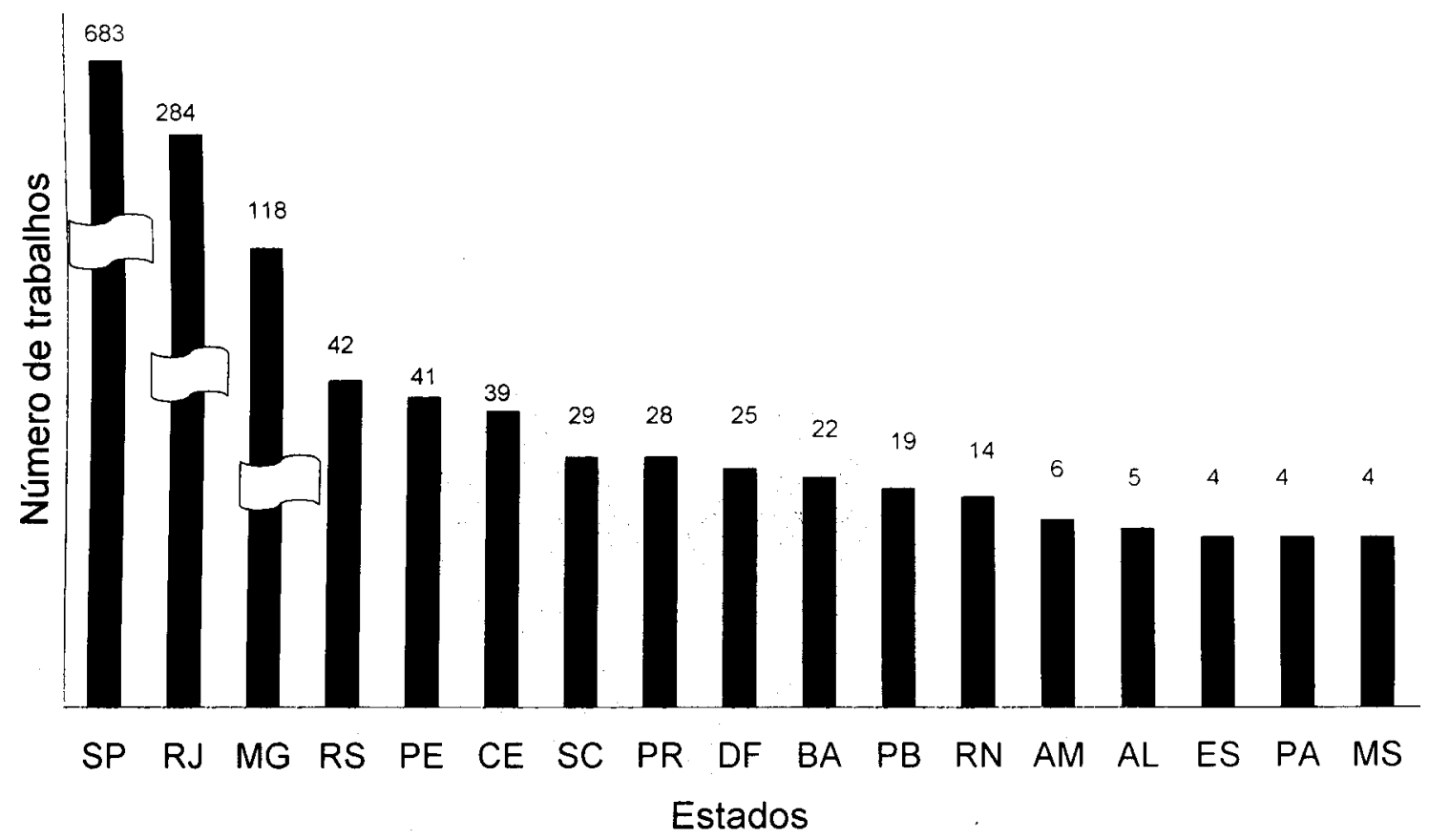

Figura 3. Distribuição dos trabalhos publicados por Estado (1978-1997). 
Tabela 4. Distribuição dos trabalhos publicados por origem: Instituições.

\begin{tabular}{|c|c|c|c|c|c|c|c|c|c|c|c|c|c|c|c|c|c|c|c|c|c|}
\hline $\begin{array}{l}\text { Ano } \\
\text { Instituição }\end{array}$ & 78 & 79 & 80 & 81 & 82 & 83 & 84 & 85 & 86 & 87 & 88 & 89 & 90 & 91 & 92 & 93 & 94 & 95 & 96 & 97 & Total \\
\hline USP & 16 & 12 & 5 & 4 & 5 & 8 & 15 & 7 & 6 & 16 & 14 & 17 & 19 & 18 & 31 & 28 & 17 & 21 & 22 & 20 & 301 \\
\hline UNICAMP & 10 & 5 & 9 & 8 & 4 & 15 & 8 & 11 & 10 & 14 & 11 & 12 & 7 & 11 & 6 & 11 & 10 & 10 & 15 & 16 & 203 \\
\hline UFRJ & 1 & 3 & 2 & 4 & 4 & 5 & 9 & 17 & 17 & 10 & 11 & 11 & 8 & 7 & 6 & 1 & 7 & 12 & 9 & 15 & 159 \\
\hline UFSCar & 1 & 3 & 2 & 4 & 5 & 4 & 3 & 5 & 7 & 2 & 7 & 3 & 3 & 4 & 2 & 6 & 7 & 7 & 6 & 3 & 84 \\
\hline UFMG & 1 & 2 & 3 & & & 2 & 2 & 2 & 1 & 2 & 8 & 6 & 7 & 5 & 1 & 3 & 13 & 3 & 4 & 10 & 75 \\
\hline UNESP & & & & 1 & & & 2 & & 1 & 6 & 3 & 7 & 7 & 3 & 6 & 1 & 5 & 7 & 6 & 5 & 60 \\
\hline UFPE & 4 & & 2 & 2 & 1 & 2 & 2 & 2 & & 1 & 9 & 1 & & 2 & & & 4 & 3 & 4 & 1 & 40 \\
\hline UFCE & 1 & 2 & & & & 1 & 2 & 6 & 5 & 4 & 2 & 6 & 1 & & 1 & 4 & 2 & 1 & 1 & & 39 \\
\hline UFF & & & & & & & & 2 & & 2 & 3 & 3 & 4 & 2 & 2 & & 1 & 4 & 3 & 3 & 29 \\
\hline UFSC & & & & & & & & & 1 & 1 & 2 & & 1 & 1 & 2 & 2 & 4 & 3 & 5 & 6 & 28 \\
\hline UFRRJ & 1 & & 1 & & & & & & 1 & 2 & 1 & & 2 & & 1 & 4 & 3 & 3 & 4 & 3 & 26 \\
\hline UFRGS & & 2 & 1 & 2 & & 1 & & 1 & & 2 & & 1 & & 1 & 1 & 2 & 2 & 2 & 6 & 2 & 26 \\
\hline UFBA & & & & & & & 1 & 1 & 2 & 1 & & 1 & 1 & & 2 & 2 & 1 & 3 & 4 & 3 & 22 \\
\hline PUC - RJ & 2 & & & 1 & 1 & 1 & 1 & 2 & 1 & 1 & 1 & 2 & & 1 & 2 & & & 1 & 2 & 1 & 20 \\
\hline UFPB & & & & & & & & & 2 & & & 3 & 1 & 3 & & 3 & 1 & 1 & 2 & 3 & 19 \\
\hline UFV & & & & & & & 1 & 1 & & & & 2 & & & & 1 & 3 & 2 & 4 & 4 & 18 \\
\hline UnB & & 1 & & & 1 & & 1 & 1 & & & & 1 & & 1 & 1 & 1 & 2 & 2 & 1 & 3 & 16 \\
\hline UFSM & & 1 & & & & & & & & & 3 & 1 & 1 & & 1 & 2 & 2 & & 2 & 3 & 16 \\
\hline UFPR & & & & & 1 & & & & & & 1 & 1 & 1 & 2 & & 2 & 1 & 2 & 2 & 2 & 15 \\
\hline UFRN & & & 1 & & & 2 & 3 & 1 & 2 & 1 & & 1 & & & & & 1 & 1 & 1 & & 14 \\
\hline IME - RJ & & & & & 1 & 1 & 1 & 1 & & 1 & 1 & 1 & 2 & & & & & 1 & 1 & & 11 \\
\hline CNEN - RJ & & 2 & & & & & & & 1 & 4 & & & & & & & & 1 & 3 & & 11 \\
\hline CTA - SP & & 1 & & & & & & 1 & 2 & & 1 & & & 1 & & & 1 & 1 & 1 & 1 & 10 \\
\hline CBPF - RJ & 1 & & & & & & 1 & 1 & & & 5 & & & & & & 1 & & & 1 & 10 \\
\hline UFU & & & & & & & & & & 1 & 2 & & & & & 1 & 2 & & 1 & & 9 \\
\hline CNPq - DF & & 1 & & 1 & & 1 & & 1 & 1 & 1 & 1 & & & 1 & & 1 & & & & & 9 \\
\hline UFOP & & & & & & & & & & & & & & & & 4 & 2 & 1 & & 1 & 8 \\
\hline UEM & & & 1 & & & & & 1 & & & & & & & 1 & 1 & & 1 & 1 & 1 & 7 \\
\hline UFAL & & & & & 2 & & & & 1 & & & & & & & 1 & & & & 1 & 5 \\
\hline INPA - AM & & & & & & 1 & & & & 1 & & & 2 & & & & & 1 & & & 5 \\
\hline PUC & & & & & & & & & & & & & & & & 1 & & & 1 & 2 & 4 \\
\hline Esc. Téc. Fed. & & & & & & & & & & & & & & & 2 & & 1 & & 1 & 4 & \\
\hline UFJF & & & & & & & & & & & & 1 & & & & & & & 2 & 1 & 4 \\
\hline UFES & & & & & & & & & & & & & & & & 1 & 1 & & 1 & 1 & 4 \\
\hline UFMS & & & & & & & & & & & & & & & 1 & & 1 & & & 2 & 4 \\
\hline EXTERIOR & & & & & & & & & & & & & & & & & & & & & 179 \\
\hline OUTROS* & & & & & & & & & & & & & & & & & & & & & 59 \\
\hline
\end{tabular}

*Instituições que contribuíram com: um artigo - Instituto Botânico (90), IPEN (89), EPM (90), FUNDACENTRO (91), CETESB (92), UNAERP (92), UNIVALE (94), UNIMEP (96), Instituto Adolfo Lutz (97), CEPEL (85), Observatório Nacional - CNPq (94), Ind. Nucleares Brasileiras (96), UERG (97), UNIRIO (97), USIMINAS (89), ESAL (79), UFPA (87), UFAM (90), UFRPE (97), UE Pta. Grossa (95), UNIVALI (96), UNIR (96) e UFGO (95); dois artigos - INPE (88, 93), FAENQUIL (92, 95), EMBRAPA - S. Carlos (96), Petrobrás $(84,92)$, INT $(91,96)$, FIOCRUZ (92,95), UENF $(95,96)$, FIOCRUZ (90, 94), UFSE (88, 95), IAP Londrina (92), UFRR (96, 97); três artigos - Inst. Butantã (88, 91, 92), Inst. Biológico (91, 95, 97), UEL (90, 93), UFPI (87, 96, 97), UFMT (92, 94, 96), Museu Emílio Goeldi - PA $(87,90)$. 
Tabela 5. Distribuição dos trabalhos publicados por origem: Exterior.

\begin{tabular}{lclc}
\hline País & Número de Trabalhos & País & Número de Trabalhos \\
\hline França & 25 & Canadá & 6 \\
USA & 24 & Paquistão & 4 \\
Portugal & 21 & Bélgica & 3 \\
Inglaterra & 18 & Suiça & 3 \\
Alemanha & 17 & Colômbia & 2 \\
Argentina & 16 & Polônia & 2 \\
Cuba & 13 & Venezuela & 2 \\
Chile & 8 & Outros* & 8 \\
Itália & 7 & TOTAL & 179
\end{tabular}

*Países que contribuíram com um artigo: Suécia, Costa Rica, Japão, México, Dinamarca, Tchecoslováquia, Finlândia e Espanha.

Tabela 6. Distribuição dos trabalhos publicados pelas unidades da USP, UNICAMP, UFRJ e UNESP.

\begin{tabular}{ccc}
\hline Universidade & Unidade & Número de Trabalhos \\
\hline USP & IQ - S. Paulo & 164 \\
$(301)$ & IQ - S. Carlos & 51 \\
& DQ - FFCL - R. Preto & 38 \\
& CENA - Piracicaba & 28 \\
& Outras & 20 \\
UNICAMP & IQ & 190 \\
$(203)$ & Outras & 13 \\
UFRJ & IQ & 96 \\
$(159)$ & NPPN & 37 \\
& FF & 16 \\
& Outras & 10 \\
UNESP & IQ - Araraquara & 44 \\
$(60)$ & Outras & 16 \\
\hline
\end{tabular}

É importante destacar, neste momento, o papel da FAPESP e do MCT/CNPq/FINEP (Programa de Apoio a Publicações Científicas), órgãos financiadores que foram e continuam sendo fundamentais para manter em dia a publicação da revista.

Neste levantamento procuramos apresentar um retrato objetivo do que tem sido a revista nestes seus 20 anos. Entretanto, os dados apresentados poderão oferecer subsídios a cada leitor para realizar sua própria análise, mais aprofundada, do perfil de Química Nova.

- Dedicamos este trabalho, com muito carinho, à Dirce M. F. Campos.

\section{REFERÊNCIAS}

1. Ferraz, H.M.C. e Pardini, V. L.; Quím. Nova 1992, 15, 104. 